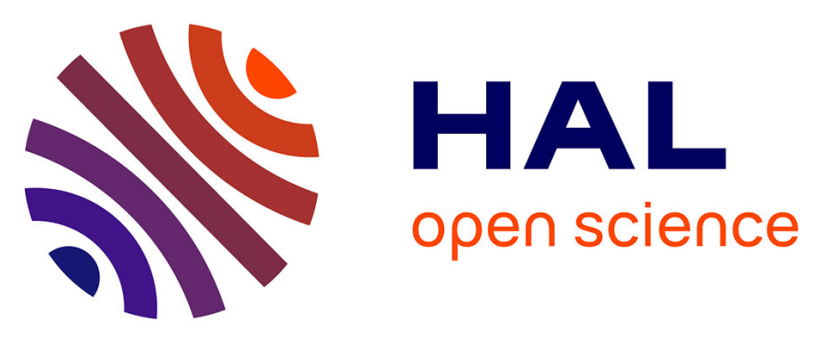

\title{
Fast multilinear Singular Values Decomposition for higher-order Hankel tensors
}

Maxime Boizard, Remy Boyer, Gérard Favier, Pascal Larzabal

\section{To cite this version:}

Maxime Boizard, Remy Boyer, Gérard Favier, Pascal Larzabal. Fast multilinear Singular Values Decomposition for higher-order Hankel tensors. IEEE Sensor Array and Multichannel Signal Processing Workshop - Invited article, Jun 2014, A Coruña, Spain. 4 p. hal-01005002

\section{HAL Id: hal-01005002 \\ https://hal-centralesupelec.archives-ouvertes.fr/hal-01005002}

Submitted on 11 Jun 2014

HAL is a multi-disciplinary open access archive for the deposit and dissemination of scientific research documents, whether they are published or not. The documents may come from teaching and research institutions in France or abroad, or from public or private research centers.
L'archive ouverte pluridisciplinaire HAL, est destinée au dépôt et à la diffusion de documents scientifiques de niveau recherche, publiés ou non, émanant des établissements d'enseignement et de recherche français ou étrangers, des laboratoires publics ou privés.

\section{(1)(1) $\$(0)$}

Distributed under a Creative Commons Attribution - NonCommercial - ShareAlikel 4.0 


\title{
Fast multilinear Singular Value Decomposition for higher-order Hankel tensors
}

\author{
Maxime Boizard*†, Rémy Boyer*, Gérard Favier ${ }^{\ddagger}$ and Pascal Larzabal ${ }^{\dagger}$ \\ ${ }^{*}$ LSS, Supelec, 3 rue Joliot-Curie F-91192 Gif-sur-Yvette, France \\ ${ }^{\dagger}$ SATIE, ENS Cachan 61, av President Wilson F-94230 Cachan, France \\ ${ }^{\ddagger}$ I3S, Université de Nice Sophia Antipolis, 2000 route des Lucioles F-06903 Sophia Antipolis, France \\ Email: maxime.boizard,pascal.larzabal@ satie.ens-cachan.fr, remy.boyer@1ss.supelec.fr, favier@i3s.unice.fr
}

\begin{abstract}
The Higher-Order Singular Value Decomposition (HOSVD) is a possible generalization of the Singular Value Decomposition (SVD) to tensors, which have been successfully applied in various domains. Unfortunately, this decomposition is computationally demanding. Indeed, the HOSVD of a $N^{t h}$. order tensor involves the computation of the SVD of $N$ matrices. Previous works have shown that it is possible to reduce the complexity of HOSVD for third-order structured tensors. These methods exploit the columns redundancy, which is present in the mode of structured tensors, especially in Hankel tensors. In this paper, we propose to extend these results to fourth order Hankel tensor. We propose two ways to extend Hankel structure to fourth order tensors. For these two types of tensors, a method to build a reordered mode is proposed, which highlights the column redundancy and we derive a fast algorithm to compute their HOSVD. Finally we show the benefit of our algorithms in terms of complexity.
\end{abstract}

\section{INTRODUCTION}

An increasing number of signal processing applications deal with multidimensional data like polarimetric STAP [1], multidimensional harmonic retrieval [2] or MIMO coding [3]. The multilinear algebra [4], [5] provides a good framework to exploit these data [6], [2] by conserving the multidimensional structure of the information. Nevertheless, generalizing matrixbased algorithms to the multilinear algebra framework is not a trivial task. In particular, there is no multilinear extension of the Singular Value Decomposition (SVD), having exactly the same properties as the SVD. However, two main decompositions exist: Canonical Polyadic Decomposition (CPD) [7], which conserves the rank properties of SVD and the uniqueness properties, and the Higher Order Singular Value Decomposition (HOSVD) [5], which keeps the orthogonality properties. HOSVD has been successfully applied in many fields and/or applications such as image processing [8], ESPRIT [2], ICA [9] and video compression [10].

In [5], it has been shown that the HOSVD of a $N^{t h}$ order tensor involves the computation of the SVD of $N$ matrix unfoldings. As a consequence, the computational cost of this algorithm is very high. In [11], it has been shown that the complexity of this algorithm can be reduced for third order structured tensors. These methods exploit the columns

The authors wish to thank DIGITEO for its financial support on the project DETMOTS-2A.

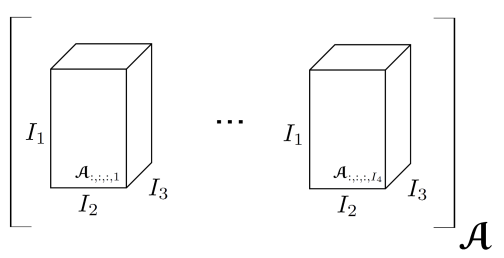

Figure 1. Representation of a fourth-order tensor with $I_{4}$ third-order tensors.

redundancy, which is present in the mode of structured tensors, including Hankel tensors. In particular, they require to determine the column redundancy. This information can be obtained through the building of a specific reordered tensor unfolding.

Hankel tensors occur naturally in signal processing applications, such as the harmonic retrieval problem [12], [13] or in the context of geoscience [14]. In this paper, we propose to extend previous results [15] to fourth order Hankel tensors. We propose two ways to extend Hankel structure to fourth order tensors. For each, a method to build a reordered tensor unfolding is proposed in order to highlight the column redundancy. Then, we derive a fast algorithm to compute their HOSVD. Finally we show the benefit of our algorithms in terms of complexity.

The following convention is adopted: scalars are denoted as italic letters, vectors as lower-case bold-face letters, matrices as bold-face capitals, and tensors are written as bold-face calligraphic letters. We use the superscripts ${ }^{H}$, for Hermitian transposition and ${ }^{*}$, for complex conjugation. A permutation of four elements is denoted $\pi=\left(\pi_{1}, \pi_{2}, \pi_{3}, \pi_{4}\right)$, the inverse permutation is denoted $\pi^{-1}$.

\section{Preliminaries in MUltilinear Algebra}

Let us consider a fourth-order tensor $\mathcal{A} \in \mathbb{C}^{I_{1} \times I_{2} \times I_{3} \times I_{4}}$ and $\mathcal{A}_{i_{1}, i_{2}, i_{3}, i_{4}}$ its elements. The 4 -mode tensor slices of $\mathcal{A}$ (obtained by fixing one index) are denoted $\mathcal{A}_{:,,,,, i_{4}} \in \mathbb{C}^{I_{1} \times I_{2} \times I_{3}}$ (in this case the fourth index is fixed to $i_{4}$ ). We recall that a fourth-order tensor can be written as the concatenation of the third-order tensors $\mathcal{A}_{:,:,:, i_{4}}$, for $i_{4} \in\left\{1 \ldots I_{4}\right\}$ (see figure 1).

\section{A. HOSVD}

One of the extensions of the SVD to the tensor case is given by the HOSVD [5]. A tensor $\mathcal{A}$ can be decomposed as 
follows:

$$
\mathcal{A}=\mathcal{K} \times{ }_{1} \mathbf{U}^{(1)} \times{ }_{2} \mathbf{U}^{(2)} \times{ }_{3} \mathbf{U}^{(3)} \times{ }_{4} \mathbf{U}^{(4)},
$$

where $\forall n, \mathbf{U}^{(n)} \in \mathbb{C}^{I_{n} \times I_{n}}$ is a unitary matrix and where $\mathcal{K} \in \mathbb{C}^{I_{1} \times I_{2} \times I_{3} \times I_{4}}$ is the core tensor, which satisfies the all-orthogonality conditions [5]. The matrix $\mathbf{U}^{(n)}$ is given by the left singular matrix of the $n^{\text {th }}$-dimension matrix unfolding (also called $n$-mode), $[\mathcal{A}]_{n}$.

Thus, the calculation of the HOSVD of a fourth-order tensor requires the computation of the left factor in the full SVD of each $[\mathcal{A}]_{n}$. However, in many applications, a truncated HOSVD is sufficient, which means that we compute only the $r_{n}$ first columns of the matrix $\mathbf{U}^{(n)}$ (where $r_{n}=\operatorname{rank}\left([\mathcal{A}]_{n}\right.$ ) is the $n$ rank of $\mathcal{A}$ ). The computational cost of the full and rank-truncated HOSVD is summarized in table I.

Table I

HOSVD FOR UNSTRUCTURED TENSORS, $I=\frac{1}{4}\left(I_{1}+I_{2}+I_{3}+I_{4}\right)$,

$$
R=\frac{1}{4}\left(r_{1}+r_{2}+r_{3}+r_{4}\right)
$$

\begin{tabular}{|c|c|c|}
\hline Operation & \multicolumn{2}{|c|}{ Cost per iteration } \\
\hline & Full HOSVD & Truncated HOSVD \\
\hline SVD of $[\mathcal{A}]_{1}$ & $4 I_{1}^{2} I_{2} I_{3} I_{4}$ & $4 r_{1} I_{1} I_{2} I_{3} I_{4}$ \\
\hline SVD of $[\mathcal{A}]_{2}$ & $4 I_{2}^{2} I_{1} I_{3} I_{4}$ & $4 r_{2} I_{1} I_{2} I_{3} I_{4}$ \\
\hline SVD of $[\mathcal{A}]_{3}$ & $4 I_{3}^{2} I_{1} I_{2} I_{4}$ & $4 r_{3} I_{1} I_{2} I_{3} I_{4}$ \\
\hline SVD of $[\mathcal{A}]_{4}$ & $4 I_{4}^{2} I_{1} I_{2} I_{3}$ & $4 r_{4} I_{1} I_{2} I_{3} I_{4}$ \\
\hline Global cost & $16 I I_{1} I_{2} I_{3} I_{4}$ & $16 R I_{1} I_{2} I_{3} I_{4}$ \\
\hline
\end{tabular}

\section{B. Structured tensors}

Definition 2.1 (Hankel tensors): A Hankel tensor is a structured tensor whose coefficients $\mathcal{A}_{i_{1}, i_{2}, i_{3}, i_{4}}$ depend only on $i_{1}+i_{2}+i_{3}+i_{4}$. In other words the coefficients can be written as $\mathcal{A}_{i_{1}, i_{2}, i_{3}, i_{4}}=a_{i_{1}+i_{2}+i_{3}+i_{4}}$.

Example 2.1 (Harmonic retrieval : single-channel case):

Let us consider $N$ samples of a times series $x_{n}$, $n \in\{1, \ldots, N\}$ modeled as a finite sum of $K$ exponentially damped complex sinusoids [16]

$$
x_{n}=\sum_{p=1}^{P} a_{p} e^{j \phi_{p}} e^{\left(-\alpha_{p}+j \omega_{p}\right) t_{n}}
$$

where the $a_{p}$ are the amplitudes, $\phi_{p}$ the phases, $\alpha_{p}$ the damping factors and $\omega_{p}$ the pulsations. $t_{n}=n \Delta t$ is the time lapse between the time origin and the sample $x_{n}$ and $\Delta t$ is the sampling time interval. For example, this type of data can model a sum of signals in one channel [16]. These data may be arranged in a fourth-order tensor $\mathcal{X} \in \mathbb{C}^{I_{1} \times I_{2} \times I_{3} \times I_{4}}$

$$
\boldsymbol{x}_{i_{1}+1, i_{2}+1, i_{3}+1, i_{4}+1}=x_{i_{1}+i_{2}+i_{3}+i_{4}-4},
$$

under the constraint that $I_{1}+I_{2}+I_{3}+I_{4}=N+3$ According to this definition, $X$ is Hankel. The HOSVD of $X$ is useful in order to estimate the pulsations with methods such as ESPRIT [2].

Definition 2.2 (Block-Hankel tensors): A block-Hankel tensor is a structured tensor whose 4-mode tensor slices (for a given dimension) $\mathcal{A}_{:,,,:, i_{4}}$ are Hankel, which means the coefficient can be written as $\mathcal{A}_{i_{1}, i_{2}, i_{3}, i_{4}}=a_{i_{1}+i_{2}+i_{3}}^{\left(i_{4}\right)}$.

Example 2.2 (Harmonic retrieval : multi-channel case): We consider the same case as example 2.1 with several channels $x_{n}^{(q)}, q \in\{1 \ldots Q\}$

$$
x_{n}^{(q)}=\sum_{p=1}^{P} a_{p}^{(q)} e^{j \phi_{p}^{(q)}} e^{\left(-\alpha_{p}+j \omega_{p}\right) t_{n}}
$$

Each channel can be folded as third-order tensor [13]

$$
x_{i_{1}+1, i_{2}+1, i_{3}+1}^{(q)}=x_{i_{1}+i_{2}+i_{3}-3}^{(q)},
$$

under the constraint that $I_{1}+I_{2}+I_{3}+=N+2$. Note that $\boldsymbol{X}^{(q)}$ is a Hankel tensor for all $q$. Finally the tensor $\boldsymbol{y} \in \mathbb{C}^{I_{1} \times I_{2} \times I_{3} \times Q}$, which contains the contribution of all channels is built concatenating the $Q$ tensors $\boldsymbol{X}^{(q)}$ :

$$
\boldsymbol{y}_{:,,,:, q}=\boldsymbol{x}^{(q)} .
$$

This tensor has a block-Hankel structure. Its HOSVD is needed in order to estimate the pulsations $\omega_{p}$.

\section{REORDERED TENSOR UNFOLDING}

\section{A. Oblique submatrices of a tensor}

In order to derive fast algorithms, it is necessary to define a reordered mode which exploits the structure of the tensors. In this way, we propose to extend the notion of oblique submatrices introduced in [11] to fourth-order tensors.

Definition 3.1 (Oblique submatrices of a tensor): For any permutation $\pi$ the oblique submatrices of a tensor $\mathcal{A}$ are defined as follows. For all $k \in\left\{0, \ldots, I_{\pi_{2}}+I_{\pi_{3}}-2\right\}$ let

$$
J^{(\pi)}(k)=\min \left(I_{\pi_{2}}, I_{\pi_{3}}, 1+k, I_{\pi_{2}}+I_{\pi_{3}}-1-k\right) .
$$

For all $i_{\pi_{4}} \in\left\{1 \ldots I_{\pi_{4}}\right\}$, the coefficients of the $I_{\pi_{1}} \times J^{(\pi)}(k)$ oblique submatrix of $\mathcal{A}$ are

$$
\mathbf{R}_{k, i_{4}}^{(\pi)}(i, j)=\mathcal{A}_{\pi^{-1}\left(i, \max \left(k-I_{\pi_{3}}, 1\right)+j-1, \min \left(k, I_{\pi_{3}}\right)-j+1, i_{\pi_{4}}\right)}
$$

An example of these matrices is shown on figure 2 .

This definition is linked to the type- 2 submatrices of a $3^{\text {rd }}$ order tensor introduced in [11] : for each $i_{\pi_{4}}$ the matrices $\mathbf{R}_{k, i_{\pi_{4}}}^{(\pi)}$ are the oblique submatrices of the $3^{\text {rd }}$-order tensor $\mathcal{A}_{\pi^{-1}\left(:,:,:, i_{\pi_{4}}\right)} \in \mathbb{C}^{I_{\pi_{1}} \times I_{\pi_{2}} \times I_{\pi_{3}}}$.

Proposition 3.1: If $\mathcal{A}$ is a Hankel or a block Hankel tensor, then all columns of each oblique submatrix $\mathbf{R}_{k, i_{4}}^{(\pi)}$ are the same.

Proof: Hence $\mathcal{A}$ is Hankel (or block Hankel), $\mathcal{A}_{i_{1}, i_{2}, i_{3}, i_{4}}$ is of the form $\mathcal{A}_{i_{1}, i_{2}, i_{3}, i_{4}}=a_{i_{1}+i_{2}+i_{3}+i_{4}}$ (or $\mathcal{A}_{i_{1}, i_{2}, i_{3}, i_{4}}=$ $a_{i_{1}+i_{2}+i_{3}}^{\left(i_{4}\right)}$ ). Then $\mathbf{R}_{k, i_{4}}^{(\pi)}(i, j)=a_{\left(i+\max \left(k-I_{\pi_{3}}, 1\right)+\min \left(k, I_{\pi_{3}}\right)+i_{4}\right.}$ (or $\mathbf{R}_{k, i_{4}}^{(\pi)}(i, j)=a_{\left(i+\max \left(k-I_{\pi_{3}}, 1\right)+\min \left(k, I_{\pi_{3}}\right)\right.}^{\left(i_{4}\right)}$ ) which does not depend on $j$.

\section{B. Reordered tensor unfolding}

Using the previous definition of oblique submatrices, it is then possible to define a reordered tensor unfolding, which highlights the columns redundancy of Hankel (and block Hankel) tensors.

Definition 3.2 (Reordered tensor unfolding): For a tensor $\mathcal{A}$, the reordered tensor unfolding along the $\pi_{1}$ dimension, 


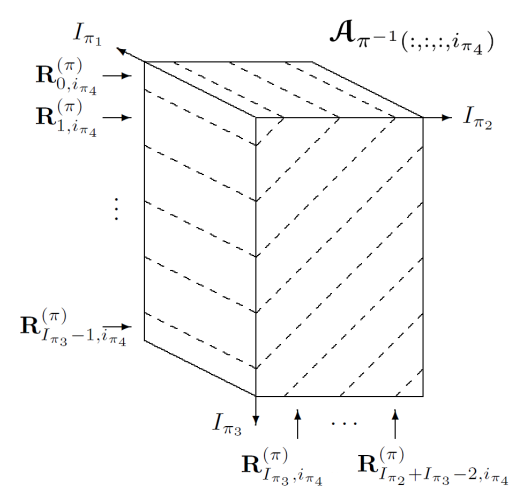

Figure 2. Representation of oblique submatrices of the 4-mode tensor slice $\mathcal{A}_{\pi^{-1}\left(:,:,:, i_{\pi_{4}}\right)}$

denoted $[\mathcal{A}]_{\pi_{1}}^{\prime}$ is defined by concatenating all the oblique submatrices for a given permutation $\pi$. In other words, the matrix $[\mathcal{A}]_{\pi_{1}}^{\prime}$ contains all matrices $\mathbf{R}_{k, i_{4}}^{(\pi)}$ for all $k \in\left\{0, \ldots, I_{\pi_{2}}+\right.$ $\left.I_{\pi_{3}}-2\right\}, i_{\pi_{4}} \in\left\{1 \ldots I_{\pi_{4}}\right\}$.

For each value of $i_{\pi_{4}}$, the set of $\mathbf{R}_{k, i_{4}}^{(\pi)}$ (for all $k \in$ $\left.\left\{0, \ldots, I_{\pi_{2}}+I_{\pi_{3}}-2\right\}\right)$ contains all the columns of $\left[\mathcal{A}_{\pi^{-1}\left(:,:,:, i_{\pi_{4}}\right)}\right]_{\pi_{1}}$ (see [11] for details). Then it is clear that $[\mathcal{A}]_{\pi_{1}}^{\prime}$ contains the same columns as $[\mathcal{A}]_{\pi_{1}}$. Therefore the left singular factor in the SVD of $[\mathcal{A}]_{\pi_{1}}^{\prime}$ is the same as in the SVD of $[\mathcal{A}]_{\pi_{1}}$.

Example 3.1: Consider the $2 \times 3 \times 3 \times 2$ Hankel tensor given by $\mathcal{A}_{i_{1}, i_{2}, i_{3}, i_{4}}=i_{1}+i_{2}+i_{3}+i_{4}$. The classic 1 -mode is given by:

$$
[\mathcal{A}]_{1}=\left[\left[\mathcal{A}_{:,:,:, 1}\right]_{1}\left[\mathcal{A}_{:,:,:, 2}\right]_{1}\right]
$$

with

$$
\left[\mathcal{A}_{:,:,:, 1}\right]_{1}=\left[\begin{array}{lllllllll}
4 & 5 & 6 & 5 & 6 & 7 & 6 & 7 & 8 \\
5 & 6 & 7 & 6 & 7 & 8 & 7 & 8 & 9
\end{array}\right]
$$

and

$$
\left[\mathcal{A}_{:,:,:, 2}\right]_{1}=\left[\begin{array}{ccccccccc}
5 & 6 & 7 & 6 & 7 & 8 & 7 & 8 & 9 \\
6 & 7 & 8 & 7 & 8 & 9 & 8 & 9 & 10
\end{array}\right] .
$$

The reordered 1-mode unfolding is equal to :

$$
\begin{gathered}
{[\mathcal{A}]_{1}^{\prime}=[\underbrace{\left[\begin{array}{l}
4 \\
5
\end{array}\right]}_{\mathbf{R}_{0,1}} \underbrace{\left[\begin{array}{ll}
5 & 5 \\
6 & 6
\end{array}\right]}_{\mathbf{R}_{1,1}} \underbrace{\left[\begin{array}{lll}
6 & 6 & 6 \\
7 & 7 & 7
\end{array}\right]}_{\mathbf{R}_{2,1}} \underbrace{\left[\begin{array}{ll}
7 & 7 \\
8 & 8
\end{array}\right]}_{\mathbf{R}_{3,1}} \underbrace{\left[\begin{array}{l}
8 \\
9
\end{array}\right]}_{\mathbf{R}_{4,1}}} \\
\underbrace{\left[\begin{array}{c}
5 \\
6
\end{array}\right]}_{\mathbf{R}_{0,2}} \underbrace{\left[\begin{array}{ll}
6 & 6 \\
7 & 7
\end{array}\right]}_{\mathbf{R}_{1,2}} \underbrace{\left[\begin{array}{lll}
7 & 7 & 7 \\
8 & 8 & 8
\end{array}\right]}_{\mathbf{R}_{2,2}} \underbrace{\left[\begin{array}{ll}
8 & 8 \\
9 & 9
\end{array}\right]}_{\mathbf{R}_{3,2}} \underbrace{\left[\begin{array}{c}
9 \\
10
\end{array}\right]}_{\mathbf{R}_{4,2}}]
\end{gathered}
$$

\section{FAST MULTILINEAR SVD FOR $4^{\text {th }}$ ORDER HANKEL} TENSORS

\section{A. Algorithms exploiting column-redundancy}

The fast multilinear SVD relies on algorithms exploiting column-redundancy as it was shown in [11]. Let us consider the unfolding of a tensor $\mathcal{A} \in \mathbb{C}^{I_{1} \times I_{2} \times I_{3} \times I_{4}}$ in the $n^{\text {th }}$ dimension, $[\mathcal{A}]_{n} \in \mathbb{C}^{I_{n} \times \prod_{k \neq n} I_{k}}$. We assume $[\mathcal{A}]_{n}$ contains column-redundancy.

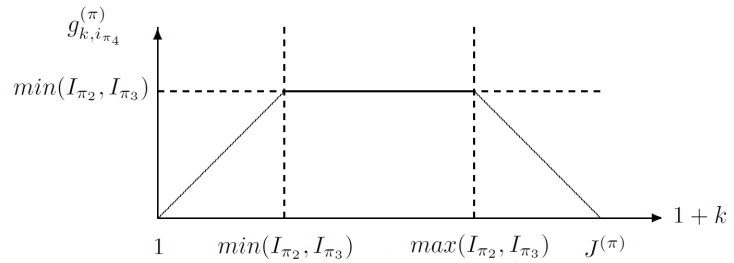

Figure 3. Number of occurrences $g_{k, i_{\pi_{4}}}^{(\pi)}$ of the columns $\mathbf{G}_{i_{\pi_{4}}}^{(\pi)}(:, k)$ for $k \in\left\{1, \ldots, J^{(\pi)}\right\}$ in the matrix $\left[\mathcal{A}_{:,:,:, i_{\pi_{4}}}\right]_{\pi_{1}}$

We define the $I_{n} \times J_{n}$ matrix $\mathbf{H}_{n}$ as the matrix obtained by removing the repeated columns in the $n$-mode $\left(J_{n} \leq\right.$ $\left.\prod_{k \neq n} I_{k}\right)$, and we denote $d_{k}^{(n)}$ the number of occurrences of the $k^{\text {th }}$ column of $\mathbf{H}_{n}$ in the $n$-mode. It is clear that :

$$
[\mathcal{A}]_{n}[\mathcal{A}]_{n}^{H}=\mathbf{H}_{n} \mathbf{D}_{n}^{2} \mathbf{H}_{n}^{H}
$$

where $\mathbf{D}_{n}=\operatorname{diag}\left(\sqrt{d_{1}^{(n)}} \cdots \sqrt{d_{J_{n}}^{(n)}}\right)$. This equality allows to prove that $\mathbf{H}_{n} \mathbf{D}_{n}$ and $[\mathcal{A}]_{n}$ have the same left factors. Thanks to the smaller dimensions of $\mathbf{H}_{n} \mathbf{D}_{n}$ it is then possible to derive fast multilinear SVD algorithms for structured tensors. However, a method need to be provided to compute $\mathbf{H}_{n}$ and $\mathbf{D}_{n}$.

\section{B. Hankel tensor}

For all $i_{4} \in\left\{1 \ldots I_{4}\right\}, k \in\left\{0, \ldots, I_{\pi_{2}}+I_{\pi_{3}}-2\right\}$ and for all permutation $\pi$, the proposition 3.1 shows that all columns of $\mathbf{R}_{k, i_{4}}^{(\pi)}$ are equal. Using this property, it is possible to derive the nonredundant matrix $\mathbf{H}_{\pi_{1}}$ and the weighting factors $d_{k}^{(\pi)}$ for the unfolding tensor $[\mathcal{A}]_{\pi_{1}}$.

First, it is possible to calculate the column redundancy for each matrix $\left[\mathcal{A}_{:,:,:, i_{\pi_{4}}}\right]_{\pi_{1}}$ (which is the 1-mode of the 4-mode tensor slice $\left.\mathcal{A}_{\pi^{-1}\left(:,:,:, i_{\pi_{4}}\right)}\right)$.

- The nonredundant matrix, denoted $\mathbf{G}_{i_{\pi_{4}}}^{(\pi)} \in \mathbb{C}^{I_{\pi_{1}} \times J^{(\pi)}}$ with $J^{(\pi)}=I_{\pi_{2}}+I_{\pi_{3}}-1$ is obtained by concatenating the first column of each matrix $\mathbf{R}_{k, i_{4}}^{(\pi)}$ for all $k \in\left\{0, \ldots, I_{\pi_{2}}+I_{\pi_{3}}-2\right\}$ and for $i_{\pi_{4}}$ set.

- The weighting factors $g_{k, i_{\pi_{4}}}^{(\pi)}$ are the number of columns of the matrices $\mathbf{R}_{k, i_{4}}^{(\pi)}$, which is equal to $J^{(\pi)}(k)$ for each $k \in\left\{0, \ldots, I_{\pi_{2}}+I_{\pi_{3}}-2\right\}$. It can be rewritten as follows (see also figure 3 ):

$$
g_{k, i_{\pi_{4}}}^{(\pi)}=\left\{\begin{array}{l}
1+k \quad \text { if } 1 \leq 1+k<\min \left(I_{\pi_{2}}, I_{\pi_{3}}\right) \\
\min \left(I_{\pi_{2}}, I_{\pi_{3}}\right) \\
\text { if } \min \left(I_{\pi_{2}}, I_{\pi_{3}}\right) \leq 1+k \leq \max \left(I_{\pi_{2}}, I_{\pi_{3}}\right) \\
J^{(\pi)}-k \quad \text { if } \max \left(I_{\pi_{2}}, I_{\pi_{3}}\right) \leq 1+k \leq J^{(\pi)}
\end{array}\right.
$$

Hence, since $J^{(\pi)}$ does not depend on $i_{\pi_{4}}$, the coefficient $g_{k, i_{\pi_{4}}}^{(\pi)}=g_{k}^{(\pi)}$ are equal for all values of $i_{\pi_{4}}$. These remarks are true for both Hankel and block Hankel tensors. However, the rest of the derivation will differ.

1) Block Hankel tensors: In this case, the matrices $\mathbf{G}_{i_{\pi_{4}}}^{(\pi)}$ are different for each values of $i_{\pi_{4}}$ (especially they have no columns in common) since $\mathbf{G}_{i_{\pi_{4}}}^{(\pi)}(i, k+1)=a_{i+k}^{\left(i_{\pi_{4}}\right)}$. Then 
the matrix $\mathbf{H}_{\pi_{1}}$ and the weighting factors $d_{k}^{(\pi)}$ are obtained by concatenating the matrices $\mathbf{G}_{i_{\pi_{4}}}^{(\pi)}$ and the weighting factors $g_{k, i_{\pi_{4}}}^{(\pi)}$ for all $i_{\pi_{4}} \in\left\{1 \ldots I_{\pi_{4}}\right\}$. Finally the dimensions of the matrix $\mathbf{H}_{\pi_{1}}$ are $I_{\pi_{1}} \times I_{\pi_{4}} J^{(\pi)}$.

2) Hankel tensors: In this case, the matrices $\mathbf{G}_{i_{\pi_{4}}}^{(\pi)}$ have some columns in common. First, the number of different columns in $[\mathcal{A}]_{\pi_{1}}^{\prime}$ is equal to $L^{(\pi)}=I_{\pi_{2}}+I_{\pi_{3}}+I_{\pi_{4}}-2$ since the values of $i_{\pi_{2}}+i_{\pi_{3}}+i_{\pi_{4}}$ are between 3 and $I_{\pi_{2}}+I_{\pi_{3}}+I_{\pi_{4}}$. The dimensions of $\mathbf{H}_{\pi_{1}}$ are then equal to $I_{\pi_{1}} \times L^{(\pi)}$.

Then the redundancy between the matrices $\mathbf{G}_{i_{\pi_{4}}}^{(\pi)}$ can be calculated, using $\mathbf{G}_{i_{\pi_{4}+1}}^{(\pi)}(i, k)=a_{i+k+i_{\pi_{4}}+1}=\mathbf{G}_{i_{\pi_{4}}}^{(\pi)}(i, k+$ 1 ). In other words, it means the matrices $\mathbf{G}_{i_{\pi_{4}}+1}$ and $\mathbf{G}_{i_{\pi_{4}}}$ can be written as follows:

$$
\begin{aligned}
\mathbf{G}_{i_{\pi_{4}}} & =\left[\mathbf{g}_{1}, \ldots, \mathbf{g}_{J^{(\pi)}}\right] \\
\mathbf{G}_{i_{\pi_{4}}+1} & =\left[\mathbf{g}_{2}, \ldots, \mathbf{g}_{J^{(\pi)}}, \mathbf{g}_{1}^{\prime}\right]
\end{aligned}
$$

Thanks to this remark, it is possible to build the non redundant matrix $\mathbf{H}_{\pi_{1}}$ for the mode $[\mathcal{A}]_{\pi_{1}}$ by concatenating the matrix $\mathbf{G}_{1}^{(\pi)}$ and the last columns of the matrices $\mathbf{G}_{i_{\pi_{4}}}^{(\pi)}$ for $i_{\pi_{4}} \in\left\{2 \ldots I_{\pi_{4}}\right\}$

$$
\mathbf{H}_{\pi_{1}}(i, l)= \begin{cases}\mathbf{G}_{1}^{(\pi)}(i, l) & \text { if } l \leq J^{(\pi)} \\ \mathbf{G}_{l-J^{(\pi)}+1}^{(\pi)}\left(i, J^{(\pi)}\right) & \text { if } J^{(\pi)} \leq l \leq I_{\pi_{4}}\end{cases}
$$

We can also determine in how many matrices $\mathbf{G}_{i_{\pi_{4}}}^{(\pi)}$ appears each column of $\mathbf{H}_{\pi_{1}}$ :

$$
r_{l}=\left\{\begin{array}{l}
l \quad \text { if } 1 \leq l<\min \left(I_{\pi_{4}}, J^{(\pi)}\right) \\
\min \left(I_{\pi_{4}}, J^{(\pi)}\right) \\
\text { if } \left.\min \left(I_{\pi_{4}}, J^{(\pi)}\right) \leq l \leq L^{(\pi)}-\min \left(I_{\pi_{4}}, J^{(\pi)}\right)\right) \\
L^{(\pi)}-l \text { else }
\end{array}\right.
$$

Finally the factors $d_{l}^{(\pi)}$ are obtained by combining equations (18) and (14) :

$$
d_{l}^{(\pi)}=\sum_{j=\min \left(I_{\pi_{4}}, J^{(\pi)}\right)-r_{l}}^{\min \left(I_{\pi_{4}}, J^{(\pi)}\right)} g_{j}^{(\pi)}
$$

3) Comparison of the complexities: Thanks to the previous results, the computation of the HOSVD of a Hankel or block Hankel relies on the SVD of $\mathbf{H}_{1} \mathbf{D}_{1}, \mathbf{H}_{2} \mathbf{D}_{2}, \mathbf{H}_{3} \mathbf{D}_{3}$ and $\mathbf{H}_{4} \mathbf{D}_{4}$. The costs of these fast algorithms are summarized in table II. We notice that this method allows a reduction of the complexity of one order of magnitude for Hankel block tensors and two orders of magnitude for Hankel tensors compared to the classic algorithm presented in table I.

Table II

FAST HOSVD FOR HANKEL AND BLOCK HANKEL TENSORS.

\begin{tabular}{|c|c|c|}
\hline Operation & \multicolumn{2}{|c|}{ Cost per iteration } \\
\hline & Hankel & Block Hankel \\
\hline SVD of $[\mathcal{A}]_{1}$ & $4 r_{1} I_{1}\left(I_{2}+I_{3}+I_{4}\right)$ & $4 r_{1} I_{1} I_{4}\left(I_{2}+I_{3}\right)$ \\
\hline SVD of $[\mathcal{A}]_{2}$ & $4 r_{2} I_{2}\left(I_{1}+I_{3}+I_{4}\right)$ & $4 r_{2} I_{2} I_{4}\left(I_{1}+I_{3}\right)$ \\
\hline SVD of $[\mathcal{A}]_{3}$ & $4 r_{3} I_{3}\left(I_{1}+I_{2}+I_{4}\right)$ & $4 r_{3} I_{3} I_{4}\left(I_{1}+I_{2}\right)$ \\
\hline SVD of $[\mathcal{A}]_{4}$ & $4 r_{4} I_{4}\left(I_{1}+I_{2}+I_{3}\right)$ & $4 r_{4} I_{4} I_{3}\left(I_{1}+I_{2}\right)$ \\
\hline Global cost for cube tensor & $48 R I^{2}$ & $24 R I^{3}$ \\
\hline
\end{tabular}

\section{CONClusion}

In this paper, we extended the previous results on thirdorder Hankel tensors to fourth order Hankel and block Hankel tensors. The reordered tensor unfolding, which highlights the column redundancy, has been detailed for these two types of tensors. Then a fast algorithm to compute their HOSVD has been proposed. Finally the benefit in terms of complexity of our algorithms has been evaluated: the reduction of the complexity is one order of magnitude for Hankel block tensors and two orders of magnitude for Hankel tensors. These results shows the interest of our approach. It could also be interesting to consider other fourth-order structured tensors like Hermitian or Toeplitz tensors.

\section{REFERENCES}

[1] M. Boizard, G. Ginolhac, F. Pascal, and P. Forster, "A new tool for multidimensional low-rank STAP filter: Cross HOSVDs," in Proceedings of EUSIPCO, Bucharest, Romania, 2012.

[2] M. Haardt, F. Roemer, and G. Del Galdo, "Higher-order SVD-based subspace estimation to improve the parameter estimation accuracy in multidimensionnal harmonic retrieval problems," IEEE Trans. on Proc. Sig. Proc., vol. 56, no. 7, pp. 3198-3213, July 2008.

[3] G. Favier, M.N. da Costa, A.L.F. de Almeida, and J.M.T. Romano, "Tensor space time (TST) coding for MIMO wireless communication systems," Elsevier Signal Processing, vol. 92, pp. 1079 - 1092, April 2012.

[4] T. Kolda and B. Bader, "Tensor decompositions and applications," SIAM Review, vol. 51, pp. 455 - 500, 2009.

[5] L. De Lathauwer, B. De Moor, and J. Vandewalle, "A multilinear singular value decomposition," SIAM J. Matrix Anal. Apl., vol. 24, no. 4 , pp. 1253-1278, 2000.

[6] N.D. Sidiropoulos, R. Bro, and G.B. Giannakis, "Parallel factor analysis in sensor array processing," IEEE Trans. on Proc. Sig. Proc., vol. 48 , no. 8, pp. 2377-2388, August 2000.

[7] R. A. Harshman, "Foundation of the PARAFAC procedure: Model and conditions for an explanatory multi-mode factor analysis," UCLA Working Papers in Phonetics, vol. 16, pp. 1-84, December 1970.

[8] D. Muti and S. Bourennane, "Multidimensional filtering based on a tensor approach," Elsevier Signal Processing, vol. 85, pp. 2338 - 2353, 2005.

[9] L. De Lathauwer, B. De Moor, and J. Vandewalle, "Independent component analysis and (simultaneous) third-order tensor diagonalization," IEEE Trans. on Sig. Proc., vol. 49, pp. 2262-2271, 2001.

[10] M.A.O Vasilescu and D. Terzopoulos, "Multilinear subspace analysis of image ensembles," in Proc. of the IEEE Conf. on Computer Vision and Pattern Recognition, 2003.

[11] R. Badeau and R. Boyer, "Fast multilinear singular value decomposition for structured tensors," SIAM Journal on Matrix Analysis and Applications, vol. 30, no. 3, pp. 1008-1021, 2008.

[12] Xiangqian Liu and N.D. Sidiropoulos, "Almost sure identifiability of constant modulus multidimensional harmonic retrieval," Signal Processing, IEEE Transactions on, vol. 50, no. 9, pp. 2366-2368, Sep 2002.

[13] J. M. Papy, L. De Lathauwer, and S. Van Huffel, "Exponential data fitting using multilinear algebra: the single-channel and multi-channel case," Numerical Linear Algebra with Applications, vol. 12, no. 8, pp. 809-826, 2005.

[14] S. Trickett, L. Burroughs, and A. Milton, Interpolation using Hankel tensor completion, chapter 704, pp. 3634-3638

[15] R. Boyer, "Decoupled root-MUSIC algorithm for multidimensional harmonic retrieval," in IEEE 9th Workshop on Signal Processing Advances in Wireless Communications, Recife, Brazil, 2008, pp. 16 20.

[16] P. Stoica and R. L. Moses, Spectral Analysis of Signals, Prentice Hall, 2005. 